\title{
LOCAL SMOOTHING ESTIMATES RELATED TO THE CIRCULAR MAXIMAL THEOREM
}

\author{
Wilhelm Schlag and Christopher D. Sogge
}

\section{Introduction}

In 1976 Stein [18] showed that when $n \geq 3$ the spherical maximal function

$$
\sup _{t>0} \int_{S^{n-1}}|f(x+t y)| d \sigma(y)
$$

is bounded on $L^{p}\left(\mathbb{R}^{n}\right)$ if $p>n /(n-1)$. He also showed that no such result can hold for $p \leq n /(n-1)$ if $n \geq 2$. Thus, the 2 -dimensional case is more complicated since the circular maximal operator corresponding to $n=2$ is not bounded on $L^{2}$.

Some 10 years passed before Bourgain [2] finally showed that the circular maximal function is bounded on $L^{p}\left(\mathbb{R}^{2}\right)$ for every $2<p \leq \infty$. Somewhat later this result was extended to variable coefficients under the assumption of cinematic curvature by Sogge [13] and then a somewhat stronger result involving local smoothing estimates was obtained by Mockenhaupt Seeger and Sogge in [9] and [10].

These local smoothing estimates, as well as Bourgain's original techniques, can be used to show that if one modifies the definition so that the supremum is taken over, say, $1 \leq t \leq 2$ then the resulting circular maximal function is actually bounded from $L^{p}\left(\mathbb{R}^{2}\right)$ to $L^{q}\left(\mathbb{R}^{2}\right)$ for some $q>p$ depending on $p>2$. In either case, though, it seems certain that the techniques in [2] or [9] will not give sharp estimates of this type. Recently, though, Schlag [11] obtained bounds which are of the best possible nature. Specifically, if we set

$$
\mathcal{M} f(x)=\sup _{1 \leq t \leq 2} \int_{S^{1}}|f(x+t y)| d \sigma(y)
$$

then $\mathcal{M}: L^{p}\left(\mathbb{R}^{2}\right) \rightarrow L^{q}\left(\mathbb{R}^{2}\right)$ if $(1 / p, 1 / q)$ lies in the interior of the triangle $\mathcal{T}$ with vertices $(2 / 5,1 / 5),(1 / 2,1 / 2)$ and $(0,0)$. In view of Bourgain's theorem it is also of course bounded when the exponents lie on the half open line connecting

\footnotetext{
Received March 15, 1996.

The authors were supported in part by the National Science Foundation. While the research was carried out the first author was at the California Insitute of Technology and the second author was at the University of California, Los Angeles.
} 
$(1 / 2,1 / 2)$ and $(0,0)$. Schlag also showed that, except possibly for endpoints, this result is sharp (cf. $\S 4$ for similar arguments). The positive results were obtained using the "combinatorial method" of Kolasa and Wolff [7].

Because of the earlier estimates in [2], [9] and [10], the key point was to establish favorable bounds for operators related to $\mathcal{M}$ near $L^{5 / 2} \rightarrow L^{5}$. Our main result also involves these spaces. Specifically, if we let

$$
\left(A_{\alpha} f\right)(t, x)=\int_{\mathbb{R}^{2}} e^{i x \cdot \xi+i t|\xi|} \hat{f}(\xi) d \xi /(1+|\xi|)^{\alpha},
$$

then we have the following

Theorem 1.1. Suppose that $1 \leq p \leq 5 / 2$ and that $q=3 p^{\prime}$, where $p^{\prime}=p /(p-1)$. Then

$$
\left\|A_{\alpha} f\right\|_{L^{q}\left([1,2] \times \mathbb{R}^{2}\right)} \leq C_{\alpha}\|f\|_{L^{p}\left(\mathbb{R}^{2}\right)}, \quad \alpha>6(1 / 4-1 / q) .
$$

This estimate can be used to give a different proof of Schlag's theorem. Indeed, if one interpolates with the $L^{p} \rightarrow L^{p}$ local smoothing estimates in [9], then one sees that

$$
\left\|A_{\alpha} f\right\|_{L^{q}\left([1,2] \times \mathbb{R}^{2}\right)} \leq C_{\alpha}\|f\|_{L^{p}\left(\mathbb{R}^{2}\right)}
$$

for some $\alpha<1 / 2-1 / q$ if $(1 / p, 1 / q)$ is in the interior of the triangle $\mathcal{T}$ mentioned before. Because of this and the fact that the circular averaging operator is basically $A_{1 / 2} f(t, x)+A_{1 / 2} f(-t, x)$, Schlag's estimate follows from the last inequality and Sobolev's lemma. (See [9], [15] for similar arguments.) Also, arguments from [13] show that, up to an endpoint, the bounds in (1.3) are of the best possible nature.

Inequality (1.3) is related to a local smoothing conjecture stated in Sogge [13] which would correspond to (1.3) when $p=q=4$. Also, (1.3) can be thought of as a generalization of estimates due to Strichartz [19] in (1+2)-dimensions. His estimate in this context is equivalent to

$$
\left\|A_{1 / 2} f\right\|_{L^{6}\left([1,2] \times \mathbb{R}^{2}\right)} \leq C\|f\|_{L^{2}\left(\mathbb{R}^{2}\right)},
$$

which, up to an endpoint, agrees with (1.3) when $p=2$. The dual version of (1.4) turns out to be equivalent to the following restriction theorem for the Fourier transform

$$
\left(\int_{\mathbb{R}^{2}}|\hat{F}(|\xi|, \xi)|^{2} d \xi /|\xi|\right)^{1 / 2} \leq C\|F\|_{L^{6 / 5}\left(\mathbb{R}^{3}\right)}
$$

This and its version for higher-dimensions are related to the earlier $L^{2}\left(S^{n-1}\right)$ restriction theorems of Fefferman [4] and Stein and Tomas [20].

Let us now give an overview of the proof of (1.3). The basic strategy is to try to adapt the proof of the Carleson-Sjölin theorem [3], [5], [6] and the related restriction theorems for $S^{1}$ going back to Fefferman, Stein and Zygmund [4], 
[21]. As in all of these works, we exploit the fact that the exponent in the left is larger than 4 . In our case, we realize, by squaring the quantity in the norm, that it suffices to prove that

$$
\left\|T_{\alpha}(f \otimes f)\right\|_{L^{5 / 2}\left([1,2] \times \mathbb{R}^{2}\right)} \leq C_{\alpha}\|f\|_{L^{5 / 2}\left(\mathbb{R}^{2}\right)}^{2}, \quad \alpha>3 / 10,
$$

if $(f \otimes f)(x, y)=f(x) f(y)$ and

$$
T_{\alpha} G(t, x)=\iint e^{i x \cdot(\xi+\eta)+i t(|\xi|+|\eta|)} \hat{G}(\xi, \eta) \frac{d \xi d \eta}{(1+|\xi|)^{\alpha}(1+|\eta|)^{\alpha}} .
$$

The rest follows from the easy estimate corresponding to $p=1$. In practice we shall only prove dyadic versions of this inequality since they are simpler and also yield (1.3).

To obtain $\left(1.3^{\prime}\right)$ we use a slight variation of an inequality of Klainerman and Machedon [8] which is also related to the restriction theorem for $S^{1}$. To describe this, let us first remark that it would be very convenient if $T_{\alpha}: L^{2} \rightarrow L^{2}$ with $\alpha=1 / 4$. For then we would obtain $\left(1.3^{\prime}\right)$ easily using interpolation since $T_{\alpha}: L^{\infty} \rightarrow L^{\infty}$ when $\alpha>1 / 2$. Unfortunately this $L^{2}$-inequality fails. Indeed the version mentioned with $\alpha=1 / 4$ would be equivalent to $\left(1.4^{\prime}\right)$ holding with $p=6 / 5$ replaced by $p=4 / 3$ in the right (and a slightly different measure in the left). One can see that this is impossible by using a variant of Knapp's counterexample showing that there is no $L^{4 / 3}\left(\mathbb{R}^{2}\right) \rightarrow L^{2}\left(S^{1}\right)$ restriction theorem (see [20]). The sharp estimate would be that $T_{\alpha}: L^{2} \rightarrow L^{2}$ when $\alpha=3 / 8$, which is of no use for $\left(1.3^{\prime}\right)$.

Fortunately, though, there is a substitute for the inequality corresponding to $\alpha=1 / 4$. Specifically, if $\varepsilon>0$,

$$
\left\|T_{1 / 4} G\right\|_{L^{2}\left([1,2] \times \mathbb{R}^{2}\right)}^{2} \leq C_{\varepsilon} \iint\left|\hat{G}(\xi, \eta) \operatorname{angle}^{-1 / 2-\varepsilon}(\xi, \eta)\right|^{2} d \xi d \eta .
$$

Here angle $(\xi, \eta) \in[0, \pi]$ denotes the angle of the rays through the origin and $\xi$ and $\eta$, respectively. This is a slightly stronger version of an inequality of Klainerman and Machedon [8] suggested also by subsequent work of Beals and Bezard [1] and the proofs of $L^{4}$ oscillatory integral theorems in the plane where similar singular factors arise as the one in the right side of (1.5).

The singular factor complicates things; however, it turns out that we can exploit the conormality of $A_{\alpha}$ and hence $T_{\alpha}$ to get around this. To be more specific, if $\hat{G}(\xi, \eta)$ vanishes when $\xi$ and $\eta$ are outside of a small cone containing $(0,1)$ then one can strengthen the $L^{\infty}$-inequality mentioned before and obtain

$$
\left\|T_{\alpha} G\right\|_{L^{\infty}\left([1,2] \times \mathbb{R}^{2}\right)} \leq C_{\alpha} \int_{-\infty}^{\infty} \int_{-\infty x_{2}, y_{2} \in \mathbb{R}}^{\infty} \sup _{|G(x, y)| d x_{1} d y_{1}, \quad \alpha>1 / 2 .}
$$

If we split the operator dyadically with respect to the size of angle $(\xi, \eta)$ and use elementary arguments involving microlocal analysis, it turns out that we can 
see that interpolation and the last two inequalities imply that, when $\alpha>3 / 10$, the $L^{5 / 2}$-norm of $T_{\alpha} G$ is dominated by

$$
\left\|\left|x_{1}-y_{1}\right|^{-2 / 5} G(x, y)\right\|_{L_{x_{1}, y_{1}}^{5 / 3} L_{x_{2}, y_{2}}^{5 / 2}} .
$$

Here we are using the mixed-norm notation that

$$
\|H(v, w)\|_{L_{v}^{p} L_{w}^{q}}^{p}=\int\|H(v, \cdot)\|_{L^{q}}^{p} d v .
$$

If, as in the first step, we take $G(x, y)=f(x) f(y)$ we see via the HardyLittlewood inequality for fractional integrals that (1.6) is dominated by $\|f\|_{L^{5 / 2}}^{2}$ which completes the proof.

These arguments and related ones from [14] will also allow us to prove similar smoothing estimates for Fourier integral operators if, as in [13], we assume cinematic curvature and conormality. For simplicity we shall postpone dealing with these results until $\S 3$, after the simpler constant coefficient case has been treated.

Finally, in $\S 4$, we shall present some results concerning higher dimensions. Here, in particular, we shall be able to find (up to endpoints) the sharp range of Lebesgue spaces between which the spherical maximal operator is bounded.

\section{Local smoothing for circular averages}

In this section we shall prove our main inequality (1.3). The estimate for $p=1$ follows easily from stationary phase (cf. [15]), so it suffices to prove the other endpoint, that is,

$$
\left\|A_{\alpha} f\right\|_{L^{5}\left([1,2] \times \mathbb{R}^{2}\right)} \leq C_{\alpha}\|f\|_{L^{5 / 2}\left(\mathbb{R}^{2}\right)}, \quad \alpha>3 / 10,
$$

if $A_{\alpha}$ is as in (1.2).

If we choose $\beta \in C_{0}^{\infty}((1 / 2,2))$ satisfying $\sum_{-\infty}^{\infty} \beta\left(2^{k} s\right)=1, s>0$, and set

$$
A^{\lambda} f(t, x)=\int e^{i x \cdot \xi+i t|\xi|} \beta(|\xi| / \lambda) \hat{f}(\xi) d \xi
$$

then this in turn would follow from the dyadic bounds

$$
\left\|A^{\lambda} f\right\|_{L^{5}\left([1,2] \times \mathbb{R}^{2}\right)} \leq C_{\alpha} \lambda^{\alpha}\|f\|_{L^{5 / 2}\left(\mathbb{R}^{2}\right)}, \alpha>3 / 10 .
$$

Since low frequencies can be handled easily using Sobolev's theorem and elementary multiplier operator bounds, it suffices to only verify this when $\lambda$ is bigger than a fixed large constant.

If we use another partition of unity we immediately see that it suffices to only consider functions $f$ whose Fourier transform vanishes outside of a small fixed conic neighborhood of $(0,1)$. With this in mind, let us set

$$
\left(T^{\lambda} G\right)(t, x)=\iint e^{i x \cdot(\xi+\eta)+i t(|\xi|+|\eta|)} \psi_{\lambda}(\xi, \eta) \hat{G}(\xi, \eta) d \xi d \eta,
$$


where $\psi_{\lambda}(\xi, \eta)$ vanishes unless $\xi$ and $\eta$ are both in this conic neighborhood of $(0,1)$ and satisfy $|\xi|,|\eta| \in[\lambda / 2,2 \lambda]$. Also, we may assume that $D_{\xi}^{\gamma_{1}} D_{\eta}^{\gamma_{2}} \psi_{\lambda}=$ $O\left(\lambda^{-\left|\gamma_{1}\right|-\left|\gamma_{2}\right|}\right)$ for all multi-indices $\gamma_{j}$. With this notation we claim that $(2.2)$ must follow from

$$
\left\|T^{\lambda} G\right\|_{L^{5 / 2}\left([1,2] \times \mathbb{R}^{2}\right)} \leq C_{\alpha} \lambda^{\alpha}\left\||y-z|^{-2 / 5} G(y, z)\right\|_{L_{y_{1}, z_{1}}^{5 / 3} L_{y_{2}, z_{2}}^{5 / 2}}, \quad \alpha>3 / 5
$$

To verify this, we first note that if $\hat{f}$ is supported as before then $\left(A^{\lambda} f\right)^{2}=$ $T^{\lambda}(f \otimes f)$ if $\psi_{\lambda}$ is the product of an appropriate bilinear conic cutoff function times $\beta(|\xi| / \lambda) \beta(|\eta| / \lambda)$. Assuming this, we get from (2.3) that

$$
\left\|A^{\lambda} f\right\|_{L^{5}\left([1,2] \times \mathbb{R}^{2}\right)}^{2} \leq C_{\alpha} \lambda^{2 \alpha}\left\|\left|y_{1}-z_{1}\right|^{-2 / 5} f(y) f(z)\right\|_{L_{y_{1}, z_{1}}^{5 / 3} L_{y_{2}, z_{2}}^{5 / 2}}, \quad \alpha>3 / 10 .
$$

This yields (2.2) since

$$
\begin{aligned}
\| \mid y_{1}- & \left.z_{1}\right|^{-2 / 5} f(y) f(z) \|_{L_{y_{1}, z_{1}}^{5 / 3} L_{y_{2}, z_{2}}^{5 / 2}}^{5 / 3} \\
& =\int_{-\infty}^{\infty} \int_{-\infty}^{\infty}\left\|f\left(y_{1}, \cdot\right)\right\|_{L^{5 / 2}(\mathbb{R})}^{5 / 3}\left\|f\left(z_{1}, \cdot\right)\right\|_{L^{5 / 2}(\mathbb{R})}^{5 / 3} d y_{1} d z_{1} /\left|y_{1}-z_{1}\right|^{2 / 3} \\
& \leq C\left(\int_{-\infty}^{\infty}\left(\left\|f\left(y_{1}, \cdot\right)\right\|_{L^{5 / 2}}^{5 / 3}\right)^{3 / 2} d y_{1}\right)^{4 / 3}=C\|f\|_{L^{5 / 2}\left(\mathbb{R}^{2}\right)}^{10 / 3},
\end{aligned}
$$

using the Hardy-Littlewood inequality for fractional integrals in the last step.

The next step is to realize that (2.3) follows via interpolation from the following two inequalities

$$
\begin{gathered}
\left\|T^{\lambda} G\right\|_{L^{\infty}\left([1,2] \times \mathbb{R}^{2}\right)} \leq C \lambda\|G\|_{L_{y_{1}, z_{1}}^{1} L_{y_{2}, z_{2}}^{\infty}}, \\
\left\|T^{\lambda} G\right\|_{L^{2}\left([1,2] \times \mathbb{R}^{2}\right)} \leq C \lambda^{\alpha}\left\||y-z|^{-1 / 2} G(y, z)\right\|_{L^{2}\left(\mathbb{R}^{4}\right)}, \quad \alpha>1 / 2 .
\end{gathered}
$$

The first inequality is the easiest to handle. Let $K^{\lambda}$ denote the kernel of $T^{\lambda}$ and recall that the symbol of $\psi_{\lambda}$ vanishes unless $\lambda / 2 \leq|\xi|,|\eta| \leq 2 \lambda$. Because of this elementary stationary phase arguments give the uniform bounds

$$
\left|K^{\lambda}(t, x ; y, z)\right| \leq C_{N} \lambda^{3}(1+\lambda|t-| x-y||)^{-N}(1+\lambda|t-| x-z||)^{-N}
$$

(cf. [15]).

One also has the uniform bounds

$$
\left|K^{\lambda}(t, x ; y, z)\right| \leq C_{N} \lambda^{-N} \quad \text { if }\left|x_{1}-y_{1}\right|+\left|x_{1}-z_{1}\right|>1 / 2,
$$

assuming, as we are, that the symbol $\psi_{\lambda}(\xi, \eta)$ vanishes when $\xi$ or $\eta$ are outside of a sufficiently small fixed conic neighborhood of $(0,1)$. Our first estimate relies 
on the fact that $K^{\lambda}$ is essentially supported in a $\lambda^{-1}$-neighborhood of the set where both $|x-y|$ and $|x-z|$ equal $t$. The second bound relies on the fact that, if the cutoff is chosen as above, then the kernel is also supported in a small neighborhood of the set where $y=z=\left(x_{1}, x_{2}+t\right)$.

Clearly the two estimates for the kernel yield

$$
\sup _{t, x, y_{1}, z_{1}} \iint\left|K^{\lambda}(t, x ; y, z)\right| d y_{2} d z_{2} \leq C \lambda .
$$

Since this is equivalent to the desired pointwise estimate, the proof of $(2.4)$ is complete.

We still have to prove the $L^{2}$-estimate. To do this it is convenient to make a further dyadic decomposition. To this end, if $\beta \in C_{0}^{\infty}((1 / 2,2))$ is as above, let us set

$$
\psi_{\lambda, j}(\xi, \eta)=\beta\left(\lambda^{1 / 2} 2^{-j} \operatorname{angle}(\xi, \eta)\right) \psi_{\lambda}(\xi, \eta), j=1,2, \ldots
$$

We then set

$$
\left(T_{j}^{\lambda} G\right)(t, x)=\iint e^{i x \cdot(\xi+\eta)+i t(|\xi|+|\eta|)} \psi_{\lambda, j}(\xi, \eta) \hat{G}(\xi, \eta) d \xi d \eta,
$$

and define $T_{0}^{\lambda}$ by setting

$$
T_{0}^{\lambda} G=T^{\lambda} G-\sum_{j \geq 1} T_{j}^{\lambda} G
$$

Notice that the sum is finite, only involving $\leq C_{0} \log \lambda$ terms, where $C_{0}$ is a fixed constant. Notice also that

$$
\psi_{\lambda, j}(\xi, \eta)=0 \text { if angle }(\xi, \eta) \notin\left[\lambda^{-1 / 2} 2^{j-1}, \lambda^{-1 / 2} 2^{j+1}\right], j \geq 1 .
$$

With all of this in mind, we claim that (2.5) is a consequence of the following uniform estimates

$$
\left\|T_{j}^{\lambda} G\right\|_{L^{2}\left(\mathbb{R}^{3}\right)} \leq C \lambda^{1 / 2} \sqrt{\lambda^{1 / 2} 2^{-j}}\|G\|_{L^{2}\left(\mathbb{R}^{4}\right)} .
$$

To verify this claim, let us first fix $\varepsilon>0$. We then note that if $\lambda$ is large and $\psi_{\lambda, j}(\xi, \eta) \neq 0$, then $\left|\left(\nabla_{\xi}-\nabla_{\eta}\right) \varphi\right| \geq|y-z| / 2$ if $1 \leq t \leq 2$ and $|y-z| \geq 2^{j} \lambda^{-1 / 2+\varepsilon}$, with $\varphi=(x-y) \cdot \xi+(x-z) \cdot \eta+t(|\xi|+|\eta|)$ being the phase function for the kernel $K_{j}^{\lambda}$ of $T_{j}^{\lambda}$. Since $D^{\gamma} \psi_{\lambda, j}=O\left(\left(2^{j} \lambda^{1 / 2}\right)^{-|\gamma|}\right)$ routine integration by parts arguments therefore yield the following bounds for this kernel stationary phase arguments

$$
\begin{aligned}
& \sup _{(t, x) \in[1,2] \times \mathbb{R}^{2}} \int_{|y-z|>2^{j} \lambda^{-1 / 2+\varepsilon}}\left|K_{j}^{\lambda}(t, x ; y, z)\right|(1+|y-z|) d y d z \\
& +\sup _{\left\{(y, z):|y-z| \geq 2^{j} \lambda^{-1 / 2+\varepsilon}\right\}}(1+|y-z|) \int_{1}^{2} \int_{\mathbb{R}^{2}}\left|K_{j}^{\lambda}(t, x ; y, z)\right| d t d x \leq C_{N, \varepsilon} \lambda^{-N},
\end{aligned}
$$


for every $N$. Hence, if we set $G=G_{j}+\tilde{G}_{j}$ where $G_{j}(y, z)=G(y, z)$ if $|y-z| \leq$ $2^{j} \lambda^{-1 / 2+\varepsilon}$ and 0 otherwise, then Young's inequality and $\left(2.5^{\prime}\right)$ yield

$$
\begin{aligned}
\left\|T^{\lambda} G\right\|_{L^{2}} & \leq \sum_{j=0}^{C_{0} \log \lambda}\left\|T_{j}^{\lambda} G_{j}\right\|_{L^{2}}+\sum_{j=0}^{C_{0} \log \lambda}\left\|T_{j}^{\lambda} \tilde{G}_{j}\right\|_{L^{2}} \\
& \leq C \sum_{j=0}^{C_{0} \log \lambda} \lambda^{1 / 2}\left(\lambda^{1 / 2} 2^{-j}\right)^{1 / 2}\left\|G_{j}\right\|_{L^{2}}+C_{N} \lambda^{-N}\left\|(1+|y-z|)^{-1} G\right\|_{L^{2}} .
\end{aligned}
$$

From this we obtain (2.5) since the support properties of $G_{j}$ give

$$
\left(\lambda^{1 / 2} 2^{-j}\right)^{1 / 2}\left\|G_{j}(y, z)\right\|_{L^{2}} \leq C \lambda^{\varepsilon / 2}\left\||y-z|^{-1 / 2} G(y, z)\right\|_{L^{2}} .
$$

To prove $\left(2.5^{\prime}\right)$, let us first handle the terms involving $j \geq 1$. The other term $T_{0}^{\lambda}$ should be thought of as a remainder term. We shall deal with it at the end.

To obtain the bounds corresponding to $j=1,2, \ldots$, we need to make one last decomposition. If $\lambda$ and $j$ are fixed, let us define a conic partition of unity of $\mathbb{R}^{2} \backslash 0,1=\sum \chi^{\nu}(\xi)$ where the $\chi^{\nu}$ are characteristic functions of nonoverlapping sectors of aperture $\approx \lambda^{-1 / 2} 2^{j}$. If we then set

$$
\hat{G}^{\nu, \nu^{\prime}}(\xi, \eta)=\chi^{\nu}(\xi) \chi^{\nu^{\prime}}(\eta) \hat{G}(\xi, \eta)
$$

it follows from orthogonality that, for fixed $t$,

$$
\int\left|T_{j}^{\lambda} G(t, x)\right|^{2} d x \leq C \sum_{\nu, \nu^{\prime}} \int\left|T_{j}^{\lambda} G^{\nu, \nu^{\prime}}(t, x)\right|^{2} d x
$$

with $C$ being a uniform constant. Since orthogonality also gives

$$
\sum_{\nu, \nu^{\prime}}\left\|G^{\nu, \nu^{\prime}}\right\|_{L^{2}}^{2}=\|G\|_{L^{2}}^{2}
$$

we conclude that it suffices to establish the uniform bounds

$$
\left\|T_{j}^{\lambda} G^{\nu, \nu^{\prime}}\right\|_{L^{2}} \leq C \lambda^{1 / 2} \sqrt{\lambda^{1 / 2} 2^{-j}}\left\|G^{\nu, \nu^{\prime}}\right\|_{L^{2}} .
$$

We have made this last conic decomposition since we can trivially make a pointwise estimate for $T_{j}^{\lambda} G^{\nu, \nu^{\prime}}$ using Schwarz's inequality. Specifically, if we use polar coordinates $\eta=\rho \Theta, \rho>0, \Theta \in S^{1}$, then

$$
\begin{aligned}
& \left|T_{j}^{\lambda} G^{\nu, \nu^{\prime}}(t, x)\right|^{2} \leq \\
& \quad C\left(\lambda^{1 / 2} 2^{-j}\right)^{-1} \int_{S^{1}}\left|\iint e^{i x \cdot(\xi+\rho \Theta)+i t(|\xi|+\rho)} \hat{G}^{\nu, \nu^{\prime}}(\xi, \rho \Theta) \psi_{\lambda, j}(\xi, \rho \Theta) d \xi \rho d \rho\right|^{2} d \Theta .
\end{aligned}
$$


To prove (2.7) we notice that because of Plancherel's theorem and (2.6) it suffices to show that the frozen operators

$$
\left(S_{\Theta} h\right)(t, x)=\iint e^{i x \cdot(\xi+\rho \Theta)+i t(|\xi|+\rho)} h(\xi, \rho) d \xi d \rho
$$

satisfy

$$
\iint\left|S_{\Theta} h(t, x)\right|^{2} d t d x \leq C \iint\left|h(\xi, \rho) \operatorname{angle}^{-1}(\xi, \rho \Theta)\right|^{2} d \xi d \rho .
$$

For then, by integrating this inequality, we obtain

$$
\begin{aligned}
& \left\|T_{j}^{\lambda} G^{\nu, \nu^{\prime}}\right\|_{L^{2}(d t d x)}^{2} \\
& \quad \leq C\left(\lambda^{1 / 2} 2^{-j}\right)^{-1} \iiint\left|\rho \hat{G}^{\nu, \nu^{\prime}}(\xi, \rho \Theta) \psi_{\lambda, j}(\xi, \rho \Theta) \operatorname{angle}^{-1}(\xi, \rho \Theta)\right|^{2} d \xi d \rho d \Theta \\
& \quad \leq C \lambda\left(\lambda^{1 / 2} 2^{-j}\right) \iint\left|\hat{G}^{\nu, \nu^{\prime}}(\xi, \eta)\right|^{2} d \xi d \eta,
\end{aligned}
$$

using in the last step, the fact that

$$
\rho^{1 / 2} \psi_{\lambda, j}(\xi, \eta) \text { angle }{ }^{-1 / 2}(\xi, \eta)=O\left(\lambda^{1 / 2} \sqrt{\lambda^{1 / 2} 2^{-j}}\right) .
$$

Inequality $\left(2.7^{\prime}\right)$ is due to Klainerman and Machedon [8]; however, for the sake of completeness, let us indicate the proof here. The main step involves making the change of variables

$$
(\tau, \zeta)=(|\xi|+\rho, \xi+\rho \Theta)
$$

while noting that the Jacobian satisfies

$$
|d(\tau, \zeta) / d(\rho, \xi)|=|1-\Theta \cdot \xi /| \xi|| \geq c(\operatorname{angle}(\xi, \Theta))^{2},
$$

for some $c>0$. Because of this, another application of Plancherel's theorem gives

$$
\begin{aligned}
\iint\left|S_{\Theta} h(t, x)\right|^{2} d t d x & \leq C \iint|h(\xi, \rho)| d(\tau, \zeta) /\left.\left.d(\rho, \xi)\right|^{-1}\right|^{2} d \tau d \zeta \\
& =C \iint|h(\xi, \rho)| d(\tau, \zeta) /\left.\left.d(\rho, \xi)\right|^{-1 / 2}\right|^{2} d \xi d \rho .
\end{aligned}
$$

This along with the preceding inequality yields $\left(2.7^{\prime}\right)$ which completes the proof of $\left(2.5^{\prime}\right)$ when $j \geq 1$.

The remaining term $T_{0}^{\lambda}$ is easier to handle. One first realizes using orthogonality considerations as above that in order to prove $\left(2.5^{\prime}\right)$ for $j=0$, it is enough to assume that $\hat{G}(\xi, \eta)$ vanishes when $\xi$ and $\eta$ are outside of a fixed sector of aperture $\approx \lambda^{-1 / 2}$. Since the symbol of $T_{0}^{\lambda}$ vanishes when $|\eta| \geq 2 \lambda$, this assumption and Schwarz's inequality yield

$$
\left|T_{0}^{\lambda} G(t, x)\right|^{2} \leq C \lambda^{3 / 2} \int\left|\int e^{i x \cdot(\xi+\eta)+i t(|\xi|+|\eta|)} \hat{G}(\xi, \eta) d \xi\right|^{2} d \eta .
$$

From this one immediately obtains the remaining case of $\left(2.5^{\prime}\right)$ using Plancherel's theorem. 


\section{Local smoothing estimates involving cinematic curvature}

In this section we shall see how results from the last one can be extended to the variable coefficient case if, as in [13], one assumes cinematic curvature and conormality. Let us start, though, by recalling the definitions.

We shall be concerned with maximal functions involving averages over curves $\Sigma_{t, x}$ in the plane depending smoothly on $(t, x) \in[1,2] \times \mathbb{R}^{2}$. We want the $t$ dependence to be non-trivial, so, working locally, we shall assume that $\Sigma_{t, x}$ is given by

$$
\Sigma_{t, x}=\{y: \Phi(x, y)=t\}
$$

where the defining function $\Phi$ is in $C^{\infty}$. We then, as in [17], make the nondegeneracy assumption that the Monge-Ampere determinant of $\Phi$ is nonsingular:

$$
\operatorname{det}\left(\begin{array}{cc}
0 & \partial \Phi / \partial x \\
\frac{\partial \Phi}{\partial y} & \frac{\partial^{2} \Phi}{\partial x \partial y}
\end{array}\right) \neq 0 \quad \text { when } \Phi(x, y)=t
$$

This means that, if $t$ is fixed, the associated Fourier integral mapping $\mathcal{E}^{\prime}\left(\mathbb{R}^{2}\right) \rightarrow$ $\mathcal{D}^{\prime}\left(\mathbb{R}^{2}\right)$ is locally a canonical graph.

The main part of the cinematic curvature condition takes into the way the curves change with respect to the $t$-parameter. For this we need to assume that there is a function $p(t, x, \xi) \in C^{\infty}\left([1,2] \times \mathbb{R}^{2} \times \mathbb{R}^{2} \backslash 0\right)$ which is homogeneous of degree one in $\xi$ and satisfies

$$
\left\{\begin{array}{l}
p\left(\Phi(x, y), x, \Phi_{x}^{\prime}(x, y)\right) \equiv 1 \\
\text { corank } p_{\xi \xi}^{\prime \prime} \equiv 1
\end{array}\right.
$$

Assuming this, if $0 \leq a \in C_{0}^{\infty}\left(\mathbb{R}^{2} \times \mathbb{R}^{2}\right)$, we shall consider maximal functions of the form

$$
(\mathcal{M} f)(x)=\sup _{1 \leq t \leq 2} \int_{\Sigma_{t, x}}|f(y)| a(x, y) d \sigma_{t, x}(y)
$$

where $d \sigma_{t, x}$ denotes arc length measure on $\Sigma_{t, x}$. As noted in [13], the hypotheses are fulfilled when $\Sigma_{t, x}$ are geodesic circles of radius $t$ about $x$ coming from a $C^{\infty}$ Riemannian metric $g$ (with injectivity radius $>2$ ). Here, the function $p$ in (3.3) is simply $p= \pm \sqrt{\sum g^{j k} \xi_{j} \xi_{k}}$, where $g^{j k}$ is the cometric. The conormality assumption mentioned earlier just refers to the fact that the singular support, as above, is a smooth hypersurface in $[1,2] \times \mathbb{R}^{2} \times \mathbb{R}^{2}$ (see below). Also, in what follows, we of course only need to assume that our assumptions (3.2) and (3.3) hold on the support of $a$.

We can now state the main result in this section which extends Schlag's [11] constant coefficient estimates. 
Theorem 3.1. Assume that $\Sigma_{t, x}$ is as in (3.1) with $\Phi \in C^{\infty}$ satisfying (3.2) and (3.3). Then the maximal function in (3.4) satisfies

$$
\|\mathcal{M} f\|_{L^{q}\left(\mathbb{R}^{2}\right)} \leq C_{p, q}\|f\|_{L^{p}\left(\mathbb{R}^{2}\right)},
$$

if $(1 / p, 1 / q) \in \mathcal{T}$, where $\mathcal{T}$ is the interior of the triangle with vertices $(0,0)$, $(1 / 2,1 / 2)$ and $(2 / 5,1 / 5)$.

The inequality for $2<p=q \leq \infty$ was established in Sogge [13]. Also, the arguments in Schlag [11] (see also §4) show that, up to endpoints, the above results are of the best possible nature.

As in the constant coefficient case, we can obtain these estimates from a simple application of Sobolev's theorem and the appropriate smoothing estimates. To see this we need to first recall that the averaging operators in (3.4)

$$
A f(t, x)=\int_{\Sigma_{t, x}} f(y) a(x, y) d \sigma_{t, x}(y)
$$

can always be written, modulo smoothing operators which satisfy better bounds, as a finite sum of Fourier integral operators of the form

$$
\left(A_{\alpha} f\right)(t, x)=\int e^{i \phi(t, x, \xi)} a(t, x, \xi) \hat{f}(\xi) d \xi /(1+|\xi|)^{\alpha},
$$

where $\alpha=1 / 2$ and $a \in C^{\infty}\left([1,2] \times \mathbb{R}^{2} \times \mathbb{R}^{2}\right)$ vanishes for $x$ outside of a fixed compact set and satisfies

$$
\left|D_{t, x}^{\gamma_{1}} D_{\xi}^{\gamma_{2}} a(t, x, \xi)\right| \leq C_{\gamma}(1+|\xi|)^{-\left|\gamma_{2}\right|} .
$$

Also, the phases are real, in $C^{\infty}\left([1,2] \times \mathbb{R}^{2} \times \mathbb{R}^{2} \backslash 0\right)$ and homogeneous of degree one in $\xi$. If they arise from operators as in (3.5) with (3.2) holding, this will translate to the condition

$$
\operatorname{det} \partial^{2} \phi / \partial x \partial \xi \neq 0 \quad \text { on } \operatorname{supp} a \text {. }
$$

Condition (3.3) will translate to

$$
\partial \phi / \partial t=p\left(t, x, \phi_{x}^{\prime}\right), \text { corank } p_{\xi \xi}^{\prime \prime} \equiv 1 \text { on } \operatorname{supp} a .
$$

The final condition, which was automatically satisfied by the above averaging operators is the conormality condition

$$
\operatorname{corank} \phi_{\xi \xi}^{\prime \prime} \equiv 1 \text { on } \operatorname{supp} a .
$$

This ensures that the singular support of $A_{\alpha}$ is contained in the smooth hypersurface

$$
\Sigma=\left\{(t, x, y): y=\phi_{\xi}^{\prime}(t, x, \xi), \text { some } \xi \in \mathbb{R}^{2} \backslash 0\right\} .
$$

Under these hypotheses we have the following result which, along with the $L^{p} \rightarrow L^{p}$ estimates in [13] yields Theorem 3.1. 
Theorem 3.2. Assume that $A_{\alpha}$ is as in (3.6) where, as above, $a \in S_{\text {comp }}^{0}$ and the phase function satisfies $\left(3.1^{\prime}\right)-\left(3.3^{\prime}\right)$. Then if $1 \leq p \leq 5 / 2$

$$
\left\|A_{\alpha} f\right\|_{L^{3 p^{\prime}}\left([1,2] \times \mathbb{R}^{2}\right)} \leq C_{\alpha}\|f\|_{L^{p}\left(\mathbb{R}^{2}\right)}, \quad \alpha>6\left(1 / 4-1 / 3 p^{\prime}\right) .
$$

The estimate for $p=1$ follows easily from stationary phase and our conormality assumption $\left(3.1^{\prime}\right)$. Thus, to prove (3.7) it suffices, as before to show that (2.3) holds if now the squared-operator is given by

$$
\left(T^{\lambda} G\right)(t, x)=\iint e^{i \phi(t, x, \xi)+i \phi(t, x, \eta)} a_{\lambda}(t, x, \xi, \eta) \hat{G}(\xi, \eta) d \xi d \eta
$$

with

$$
a_{\lambda}=\psi_{\lambda}(\xi, \eta) a(t, x, \xi) a(t, x, \eta) .
$$

The cutoff $\psi_{\lambda}$ is as before. In particular, we recall that it vanishes when $\xi$ or $\eta$ does not belong to the intersection of a $\lambda$-annulus with a small conic neighborhood of $(0,1)$.

The last assumption implies that the direction $(0,1)$ must be transverse to the singular support of $y \rightarrow K^{\lambda}(t, x ; y)$. Given a fixed $(t, x)$ we recall that this set is contained in the smooth curve

$$
\Sigma_{t, x}=\left\{y: \phi_{\xi}^{\prime}(t, x, \xi)=y, \text { some } \xi\right\}
$$

Because of this (2.4) must hold in the present context.

Therefore, to finish matters, we just need to check that the $L^{2}$-estimate $(2.5)$ also extends to this more general setting. If we modify the definition of $T_{0}^{\lambda}$ in the obvious way we can easily adapt the constant coefficient argument to see that $\left(2.5^{\prime}\right)$ holds when $j=0$. Here one uses Schwarz's inequality and the $L^{2}$ boundedness of Fourier integral operators with phase functions satisfying $\left(3.2^{\prime}\right)$. Thus, since $(2.5)$ follows from $\left(2.5^{\prime}\right)$, we are left with verifying that the latter holds when $j \geq 1$. As before, one can use orthogonality to see that this in turn follows from $\left(2.7^{\prime}\right)$ if now

$$
\left(S_{\Theta} h\right)(t, x)=\iint e^{i \phi(t, x, \xi)+i \phi(t, x, \rho \Theta)} a_{\lambda}(t, x, \xi, \rho \Theta) h(\xi, \rho) d \xi d \rho .
$$

This inequality was essentially proved in [14] (see $\left(2.19^{\prime}\right)$ p. 1803). The argument was carried out for operators arising from solving variable-coefficient wave equations in $(1+3)$-dimensions. Since the proof only relies on the fact that the phases involved satisfy $\left(3.1^{\prime}\right)-\left(3.3^{\prime}\right)$ we conclude that $\left(2.7^{\prime}\right)$ must hold here as well, completing the proof. 


\section{Remarks on higher dimensions}

Let us conclude by showing how we can extend the earlier results to higher dimensions and find the optimal range of Lebesgue spaces between which Stein's spherical maximal function is bounded. As before, for simplicity, we shall only deal with truncated maximal operators where the supremum is taken over dilations $1 \leq t \leq 2$. This assumption can be removed if one multiplies the averaging operators by the appropriate power of $t$ so that the resulting operators are scale invariant between $L^{p}\left(\mathbb{R}^{n}\right)$ and $L^{q}\left(\mathbb{R}^{n}\right)$.

Since we can easily obtain estimates for the variable coefficient case, let us handle this situation as well. Thus, let us consider maximal functions defined by (3.4) where now $\Sigma_{t, x}$ is a smooth hypersurface in $\mathbb{R}^{n}$ given by (3.1) with $\Phi \in C^{\infty}\left(\mathbb{R}^{n} \times \mathbb{R}^{n}\right)$. We then have the following

Theorem 4.1. Let $\mathcal{M}$ be a maximal function associated with smoothly varying hypersurfaces $\Sigma_{t, x} \subset \mathbb{R}^{n}, n \geq 3$, as above. Then, if the defining function satisfies (3.2) and (3.3)

$$
\|\mathcal{M} f\|_{L^{q}\left(\mathbb{R}^{n}\right)} \leq C_{p, q}\|f\|_{L^{p}\left(\mathbb{R}^{n}\right)},
$$

provided that $(1 / p, 1 / q) \in \mathcal{Q}$, where $\mathcal{Q}$ is in the interior of the quadrangle with vertices $V_{1}=(0,0), V_{2}=((n-1) / n,(n-1) / n), V_{3}=((n-1) / n, 1 / n)$ and $V_{4}=\left(n(n-1) /\left(n^{2}+1\right),(n-1) /\left(n^{2}+1\right)\right)$. Furthermore, these results are of the best possible nature in that (4.1) can never hold if $(1 / p, 1 / q)$ is outside of the closure of $\mathcal{Q}$ and $q \geq p$.

The proof of the positive results only relies on known $L^{p}$ estimates for Fourier integral operators. We first recall that if $A f$ as in (3.5) is the averaging operator occurring in the definition of $\mathcal{M} f$, then, modulo smoothing operators, Af can be decomposed as a finite sum of operators of the form (3.6) with $\alpha=(n-1) / 2$.

If (3.2) and hence $\left(3.2^{\prime}\right)$ hold results from Seeger, Sogge and Stein [12] say that for $1<p<\infty$

$$
\left\|A_{\alpha} f(t, \cdot)\right\|_{L^{p}\left(\mathbb{R}^{n}\right)} \leq C_{q}\|f\|_{L^{p}\left(\mathbb{R}^{n}\right)}, \quad \alpha=(n-1)|1 / 2-1 / p| .
$$

The conormality assumption $\left(3.1^{\prime}\right)$ along with $\left(3.2^{\prime}\right)$ and stationary phase implies that for $1 \leq t \leq 2$ and $1 \leq p \leq 2$

$$
\left\|A_{\alpha} f(t, \cdot)\right\|_{L^{p^{\prime}\left(\mathbb{R}^{n}\right)}} \leq C\|f\|_{L^{p}\left(\mathbb{R}^{n}\right)}, \quad \alpha>(n+1)(1 / p-1 / 2) .
$$

Lastly, if $\left(3.2^{\prime}\right)$ and $\left(3.3^{\prime}\right)$ hold, the generalization of Strichartz's estimates [19] to variable coefficients in Mockenhaupt, Seeger and Sogge [10] yields

$$
\left\|A_{\alpha} f\right\|_{L^{q}\left([1,2] \times \mathbb{R}^{n}\right)} \leq C\|f\|_{L^{p}\left(\mathbb{R}^{n}\right)},
$$

provided that $2(n+1) /(n-1) \leq q<\infty$ and

$$
q=(n+1) p^{\prime} /(n-1), \alpha=(n+1) / 2-n(n+1) /(n-1) q .
$$


Notice that the fourth vertex of $\mathcal{Q}$ satisfies $q=(n+1) p^{\prime} /(n-1)$. Also, the third, as those in (4.3), lies on the line of duality. The other two, like those in (4.2), involve $p=q$. Keeping this in mind one sees that the last three inequalities and interpolation yield

$$
\left\|A_{\alpha} f\right\|_{L^{q}\left([1,2] \times \mathbb{R}^{n}\right)} \leq C_{p, q, \alpha}\|f\|_{L^{p}\left(\mathbb{R}^{n}\right)}
$$

for some $\alpha<(n-1) / 2-1 / q$ if $(1 / p, 1 / q) \in \mathcal{Q}$. Since this along with Sobolev's lemma yields (4.1) the proof is complete.

The arguments in Schlag [11] can be adapted to yield the sharpness results. For the first step, let us assume without loss of generality that the cutoff function $a$ occurring in the definition of $\mathcal{M}$ does not vanish identically on $\Sigma_{1,0}$. If then

$$
\Sigma_{1,0}^{\delta}=\left\{y \in \mathbb{R}^{n}: \operatorname{dist}\left(y, \Sigma_{1,0}\right)<\delta\right\}
$$

is a $\delta$-neighborhood of $\Sigma_{1,0}$, we conclude that for small $\delta>0$ we have uniform lower bounds of the form

$$
\int_{\left\{y \in \Sigma_{1,0}^{\delta} \cap \Sigma_{1, x}\right\}} a(x, y) d \sigma_{1, x}(y)>c_{0}>0,
$$

as long as $|x|<c_{1} \delta$ for some fixed constants $c_{0}, c_{1}>0$. Therefore, if $f_{\delta}$ denotes the characteristic function of $\Sigma_{1,0}^{\delta}$, we conclude that $\mathcal{M} f_{\delta}(x)>c_{2}>0$ for some uniform constant $c_{2}$ if $|x|<c_{1} \delta$ with $\delta$ small. Hence, for such $\delta>0$ we have uniform bounds of the form

$$
\left\|\mathcal{M} f_{\delta}\right\|_{L^{q}\left(\mathbb{R}^{n}\right)} /\left\|f_{\delta}\right\|_{L^{p}\left(\mathbb{R}^{n}\right)} \geq c_{3} \delta^{n / q-1 / p} .
$$

This implies that we must have

$$
1 / p \leq n / q \quad \text { if } \mathcal{M}: L^{p}\left(\mathbb{R}^{n}\right) \rightarrow L^{q}\left(\mathbb{R}^{n}\right) .
$$

To get another condition, we note that, after a change of coordinates, we may assume that for some $x_{0} \in \mathbb{R}^{n}$ the hypersurface $\Sigma_{1, x_{0}}$ passes through the origin with normal $(1,0, \ldots, 0)$. We may also assume that $a\left(x_{0}, 0\right) \neq 0$. Since we are assuming cinematic curvature, the equation

$$
\phi_{\xi}^{\prime}(t, \gamma(t), 1,0, \ldots, 0)=0
$$

determines a smooth curve $\gamma(t), 1 \leq t \leq 2$ satisfying $x_{0}=\gamma(1)$. If

$$
R_{\delta}=\left\{y \in \mathbb{R}^{n}: \max _{1 \leq i \leq n-1}\left|y_{i}\right|<\delta^{1 / 2},\left|y_{n}\right|<\delta\right\}
$$

then our choice of $\gamma(t)$ implies that

$$
\int_{\left\{y \in R_{\delta} \cap \Sigma_{t, x}\right\}} a(x, y) d \sigma_{t, x}(y) \geq c_{0} \delta^{(n-1) / 2}
$$


for some $c_{0}>0$ if

$$
|x-\gamma(t)|<c_{1} \delta^{1 / 2}
$$

and $t-1>\delta^{1 / 2}, c_{1}>0$ and $\delta>0$ are sufficiently small. In the case of Stein's spherical maximal function, the lower bound would be satisfied if $\max _{1 \leq i \leq n-1}\left|x_{i}\right|<c \delta^{1 / 2}$ and $1<\left|x_{n}\right|<2$ for some $c>0$. If we take $g_{\delta}=\chi_{R_{\delta}}$ then the preceding lower bound implies that for small $\delta>0$

$$
\left\|\mathcal{M} g_{\delta}\right\|_{L^{q}\left(\mathbb{R}^{n}\right)} /\left\|g_{\delta}\right\|_{L^{p}\left(\mathbb{R}^{n}\right)} \geq c_{2} \delta^{(n-1) / 2 \cdot(1+1 / q)-(n+1) / 2 p},
$$

for some positive constant $c_{2}$, from which we conclude that

$$
1 / p \leq(n-1) /(n+1) \cdot(1+1 / q) \quad \text { if } \mathcal{M}: L^{p}\left(\mathbb{R}^{n}\right) \rightarrow L^{q}\left(\mathbb{R}^{n}\right) .
$$

Finally, if make the preceding assumptions, then Stein's [18] example

$$
h(x)=|x|^{1-n} / \log |x|,|x|<1, h=0,|x| \geq 1
$$

implies that

$$
1 / p<(n-1) / n \quad \text { if } \mathcal{M}: L^{p}\left(\mathbb{R}^{n}\right) \rightarrow L^{q}\left(\mathbb{R}^{n}\right) .
$$

For $\mathcal{M} h$ is infinite on a set of positive measure under the above hypotheses and $h \in L^{p}\left(\mathbb{R}^{n}\right)$ for every $p \leq n /(n-1)$.

We conclude from (4.5)-(4.7) that if $\mathcal{M}: L^{p}\left(\mathbb{R}^{n}\right) \rightarrow L^{q}\left(\mathbb{R}^{n}\right)$ then $(1 / p, 1 / q)$ must be in the region bounded by the lines $1 / p=n / q, 1+1 / q=(n+1) /(n-1) p$ and $1 / p=(n-1) / n$. Since these along with the line $1 / p=1 / q$ determine the boundary of the quadrangle $\mathcal{Q}$ in the statement of Theorem 4.1 , the proof is complete.

\section{References}

1. M. Beals and M. Bezard, Low regularity solutions for field equations, Comm. Partial Differential Equations 21 (1996), 79-124.

2. J. Bourgain, Averages in the plane over convex curves and maximal operators, J. Anal. Math. 47 (1986), 69-85.

3. L. Carleson and P. Sjölin, Oscillatory integrals and a multiplier problem for the disk, Studia Math. 44 (1972), 287-299.

4. C. Fefferman, Inequalities for strongly singular convolution operators, Acta Math. 124 (1970), 287-299.

5. _ A note on spherical summation operators, Israel J. Math. 15 (1973), 44-52.

6. L. Hörmander, Oscillatory integrals and multipliers on $F L^{p}$, Ark. Mat. 11 (1971), 1-11.

7. L. Kolasa and T. Wolff, On some variants of the Kakeya problem, preprint.

8. S. Klainerman and M. Machedon, Space-time estimates for null forms and the local existence theorem, Comm. Pure Appl. Math. (1993), 1221-1268.

9. G. Mockenhaupt, A. Seeger and C. D. Sogge, Wave front sets, local smoothing and Bourgain's circular maximal theorem, Ann. of Math. 136 (1992), 207-218.

10. __ Local smoothing of Fourier integral operators and Carleson-Sjölin estimates, J. Amer. Math. Soc. 6 (1993), 65-130. 
11. W. Schlag, A generalization of Bourgain's circular maximal theorem, J. Amer. Math. Soc. (to appear).

12. A. Seeger, C. D. Sogge and E. M. Stein, Regularity properties of Fourier integral operators, Ann. of Math. 134 (1991), 231-251.

13. C. D. Sogge, Propagation of singularities and maximal functions in the plane, Invent. Math. 104 (1991), 349-376.

14. __ On local existence for nonlinear wave equations satisfying variable coefficient null conditions, Comm. Partial Differential Equations 18 (1993), 1795-1821.

15. __ Fourier integrals in classical analysis, Cambridge Univ. Press, Cambridge, 1993.

16. L Lectures on nonlinear wave equations, International Press, Cambridge, 1995.

17. C. D. Sogge and E. M. Stein, Averages of functions over hypersurfaces: Smoothness of generalized Radon transforms, J. Anal. Math. 54 (1990), 165-180.

18. E. M. Stein, Maximal functions: spherical means, Proc. Nat. Acad. Sci. USA 73 (1976), 2174-2175.

19. R. Strichartz, Restrictions of Fourier transforms to quadratic surfaces and decay of solutions of wave equations, Duke Math. J. 44 (1977), 705-714.

20. P. Tomas, A restriction theorem for the Fourier transform, Bull. Amer. Math. Soc. 81 (1975), 477-478.

21. A. Zygmund, On Fourier coefficients and transforms of two variables, Studia Math. 50 (1974), 189-201.

School of Mathematics, Institute for Advanced Study, Princeton, NJ 08540

E-mail address: schlag@math.ias.edu

Department of Mathematics, Johns Hopkins University, Baltimore, MD 21218

E-mail address: sogge@jhu.edu 